\title{
Controlled trial comparing the efficacy of $88 \%$ phenol versus $100 \%$ trichloracetic acid for chemical matricectomy in the management of ingrown toenail
}

\author{
Kaoutar Moustaide, Niema Aqil, Salim Gallouj, Fatima Zohra Mernissi
}

Department of Dermatology, University Hospital Hassan II, Rte SIdi Hrazem, Fes, Morroco

Corresponding author: Dr. Kaoutar Moustaide, E-mail: Moustaidek@gmail.com

\begin{abstract}
Background: The ingrown toenail results from a painful conflict between the nail plate and the adjacent soft parts. Material and methods: We conducted a prospective, comparative trial of the long-term efficacy of two methods: phenolization (88\%) and matricolysis by trichloroacetic acid (TCA) (100\%). Adult, consenting patients with ingrown toenails were alternately allocated into two treatment, to receive either $88 \%$ phenol or $100 \%$ TCA chemical matricectomy. The patients as well as the statistician were blinded to the agent being used. Post-procedure follow-up evaluated median duration of pain, discharge, and healing along with recurrence, if any, in both the groups. The group wise data was statistically analyzed. Results: On comparing the two groups, pain lasted 9.34 days in the TCA group and 18.62 days in the phenol group, this difference was not statistically significant $(\mathrm{P}=0.202)$. The tissue condition took 9.30 days to normalize in the the TCA group, while it took 17.63 days to normalize in the phenol group. This difference was found to be statistically significant $(\mathrm{P}<0.007)$. The aesthetic result and the cost of the care were similar in the 2 groups. Conclusion: Chemical matricectomy using TCA is as efficacious as phenolisation, with the advantage of faster tissue normalization.
\end{abstract}

Key words: Ingrow; Matricectomy; Phenol; Toenail; TCA

\section{INTRODUCTION}

Ingrown toenail is a widespread affection and a frequent reason for consultation in dermatology. It leads to economic losses, affecting quality of life, sporting and work activities.

Most cases require surgical management in the form of lateral partial nail avulsion of the ingrowing edge. Several surgical and non-surgical methods have been described for treating ingrown nails. however, a simple avulsion is associated with high chances of recurrence [1].

Lateral matricectomy is essential to the management of ingrown toenail. Many methods can be used to destroy the lateral matrix, this may be achieved by surgical, or more commonly, a chemical destruction. Phenol and sodium hydroxide are commonly used agents but both are frequently linked to excessive healing times [2,3]. Trichloroacetic acid (TCA) is an analogue of acetic acid. It is a well-known caustic agent extensively used in dermatology, mainly in cosmetic peelings. It causes coagulative necrosis of cells through wide protein denaturation and resultant structural cell death. It can be also used in the treatment of ingrown nail, with less postoperative drainage; however, long-term efficacy data are lacking [4].

We conducted a prospective, comparative trial of the long-term efficacy of chemical matricectomy using phenol and TCA; as well as to compare the healing times and postoperative morbidity associated with the two agents. To our knowledge, this is the first study comparing these two methods.

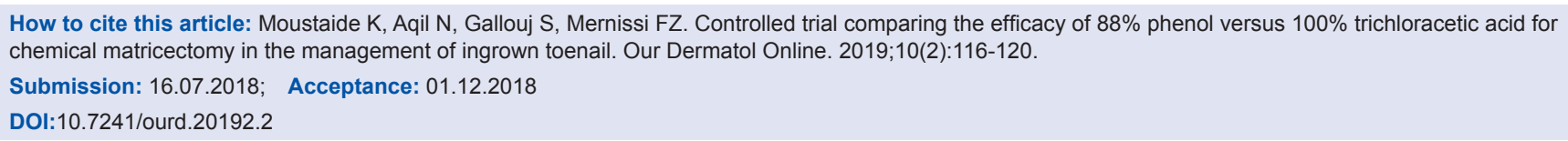




\section{METHODS}

The trial protocol was approved by the Institutional Ethical Committee of the Hassan II University Hospital. Patients, aged between 18 and 60 years, presenting to our dermatology department with a clinical diagnosis of an ingrown toenail were included. Patients with significant peripheral arterial disease, known allergy to agents being used, severe systemic disease, or conditions associated with delayed wound healing (e.g., uncontrolled diabetes mellitus) were excluded from the study.

Informed, written consent of all participants was taken prior to enrolment.

Predisposing factors were evaluated at the first visit: excessive curvature of the nail plate, toe rotation, thin nails, heavy nail folds, hyperhidrosis, bad nail clipping and the regular use of constricting footwear. Suitable antibiotic therapy was used in cases of infection.

The affected toe and foot were evaluated at baseline for the stage and type of ingrown toenail and the condition of the nail structures using the following criteria: Stage 1 was defined as the presence of only mild erythema or edema, with pain on applying pressure, Stage 2 as significant erythema or edema with sero-purulent drainage from the affected nail fold and Stage 3 as significant drainage, formation of granulation and lateral wall hypertrophy [2]. At baseline, a potassium hydroxide $(\mathrm{KOH})$ mount of the nail clipping was examined to rule out fungal infection for all patients with nail thickening, distal onycholysis, or subungual debris. Only mycologically negative cases were included in the study.

All included patients were asked to grade their pain on a visual analog scale (VAS) of 0 to 10 (0 being no pain and 10 being unbearable pain). The patients were then allocated alternately to the two treatment groups in the order in which they joined the study. Group 1 patients were treated with $88 \%$ phenol chemical matricectomy while Group 2 patients received 100\% TCA application. The allocation was done by the nursing assistant not otherwise involved in the study. The corresponding solution (phenol or TCA) was provided to the operator.

The surgical procedure consisted of digital block to anesthetize the digit, exsanguination and tourniquet application to assist hemostasis followed by partial avulsion of the lateral nail plate to remove the full length of the ingrown nail plate sliver. The lateral tunnel created was then curetted to remove any granulation tissue/crust if present.

Chemical matricectomy was then done with the appropriate agent (as per group allocation). It was provided by the operating assistant onto a cotton tipped applicator, which was vigorously rubbed onto the lateral horn of the nail matrix. The application time used for both the agents was $2 \mathrm{~min}$. Care was taken to prevent contact with surrounding structures as this could cause more extensive damage than intended and delay wound healing. Following this, the excess solution was neutralized.

The operated toe was then dressed with a paraffin gauze and bandage. Patients were instructed to rest the foot for the rest of the day and keep it in an elevated position. Analgesics were advised to be taken only when required and patients were asked to record their use. Dressings with topical antibiotics were used in all patients.

After their operations, all patients were examined at the 72-hour mark to evaluate drainage or potential complications of the surgical technique (such as hemorrhage, infection, pain or necrosis). At that point, patients measured pre-operatory and post- operatory (24 and 72 hours) pain using a visual analogic scale (from 1 to 10). Patients were then examined on day 30 (1 month), day 180 (6 months), day 270 (9 months) and day 360 (1 year), after surgery. During each visit, the wounded area was checked and any complication or sign of recurrence was noted. Bacterial cultures from the nail bed were taken in case of clinical suspicion of infection, if present, was appropriately treated.

A photographic record of each visit was maintained.

\section{RESULTS}

The study flow is outlined in (Fig. 1). SPSS version 20.0 was used to analyze the data.

A total of 120 patients were included in the study protocol (Fig. 1).

From the potential contributing factors for the disease that were assessed, we found that $55 \%$ had an excessive trimming of the lateral nail plate, $38 \%$ suffered from plantar hyperhidrosis, and 35\% showed heavy lateral nail folds., 29\% regularly used constricting footwear, 25\% 
had thin nails, $17 \%$ an excessive curvature of the nail plate and $09 \%$ a rotation of the toe (Table 1 ).

A baseline assessment of the two treatment groups is shown in (Table 1). It can be seen that both the groups were comparable with respect to their demographic characteristics and disease severity.

The intended outcome measures for the two groups are compared in (Table 2). On comparing the two groups, pain lasted 9.34 days in the TCA group and 18.62 days in the phenol group, this difference was not statistically significant $(\mathrm{P}=0.202)$. The tissue condition took 9.30 days to normalize in the the TCA group, while it took 17.63 days to normalize in the phenol group. This difference was found to be statistically significant $(\mathrm{P}<0.007)$.

Treatment was successful in all the treated patients of both the groups with resolution of all symptoms caused by the ingrown nails as noted in serial clinical photographs of patients from both groups. Four patients

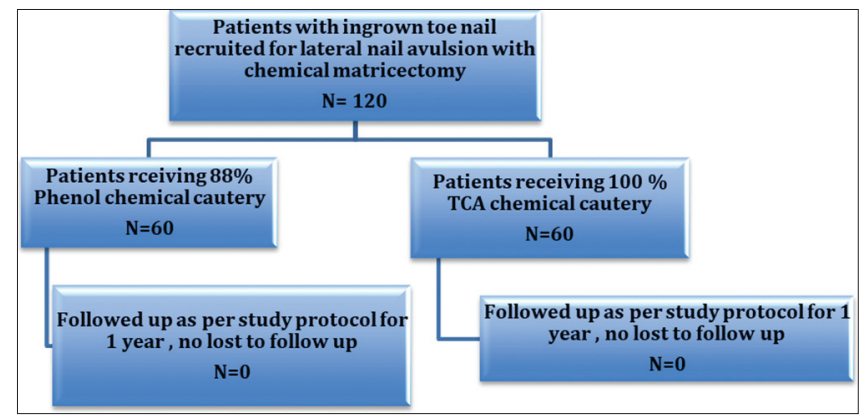

Figure 1: The study protocol.

Table 1: Baseline assessment of both treatment groups

\begin{tabular}{lcc}
\hline Baseline characterictic & Group 0 : TCA & Group 1 : Phenol \\
\hline Total number of patients(N) & 60 & 60 \\
Sexe ratio : Male / Female & $39 / 21$ & $37 / 23$ \\
Mean age (in years ) & 27 & 25,9 \\
Severity of ingrown toenail & & \\
$\quad$ Stage 1 & 08 & 08 \\
Stage 2 & 19 & 17 \\
Stage 3 & 33 & 35 \\
Mean duration of complaints & 5,2 & 9,3 \\
(in months) & & \\
\hline
\end{tabular}

Table 2 : Predisposing factors gor the ingrown nail

\begin{tabular}{ll}
\hline Excessive trimming of the lateral nail plate & $55 \%$ \\
Plantar hyperhidrosis & $38 \%$ \\
Heavy lateral nail folds & $35 \%$ \\
Use of constricting foot wear & $29 \%$ \\
Thin nails & $25 \%$ \\
Excessive curvature of the nail plate & $17 \%$ \\
Rotation of the toe & $09 \%$ \\
\hline
\end{tabular}

each in both groups were found to have secondary infection with bacteriological cultures showing Staphylococcus aureus. All the eight patients responded to standard courses of anti-staphylococcal antibiotics.

However, the rate of redicive was not related to the agent of matricolysis but was associated with the way of cutting the nails. No other side effects were recorded in any of the treatment groups.

And finally, the aesthetic result and the cost of the care were similar in the 2 groups.

\section{DISCUSSION}

Ingrown toenail is one of the most common painful nail conditions presenting to a dermatologist. It is a result of the lateral edge of the nail plate getting embedded in the nail fold (where it acts as a foreign body) resulting in a cascade of inflammation, infection and the reparative process $[2,5]$.

The condition most commonly involves the great toes and mainly affects young adults [6], as was seen in our study, where the average age was 27 and 25.9 years in both the groups. This may be related to increased chances of sustaining minor trauma to the feet owing to an active lifestyle. Rarely, elderly individuals or infants may be affected. There is no consensus on the choice of treatment for ingrown toenails.

Surgical management is the mainstay of treatment as conservative methods have a low rate of success in the long run [1].The standard surgical approach for the management of an ingrown toenail is avulsion of the nail plate with destruction of the underlying matrix $[5,6]$. The matrix destruction should be selective to minimize damage to the surrounding normal structures, but at the same time it must be complete and reliable to prevent recurrences [7].

Selective matricectomy can be performed using surgical or chemical methods. Chemical matricectomy uses various chemical agents to destroy the lateral nail matrix.

Among these, phenol is the most common agent used in most clinics. It is an effective protein denaturant which shows its cauterizing effect by producing a coagulation necrosis in the matrix and the surrounding soft tissue $[8,10]$. It has been widely studied and is reported to be more effective at preventing symptomatic recurrences (recurrence rates ranging 
from $1 \%$ to $9.6 \%$ ) as compared to nail avulsion alone $(42-83 \%)[1,11]$. However, due to the prolonged healing time of necrosed tissue, it carries an increased risk of postoperative infection [1]. The post-operative discharge generally lasts for about 2-4 weeks but may continue for up to 6 weeks [2]. In addition, phenol is contraindicated in those with moderate or severe vascular disease of the foot, conditions predisposing to delayed wound healing, allergy to the chemical and in pregnancy $[12,13]$.

In recent years, matricectomy with sodium hydroxide has been found to be as effective as phenol matricectomy, with shorter healing periods and a lower risk of local or systemic toxicity $[3,14-16]$. Sodium hydroxide causes less burns and liquefaction necrosis, resulting in less postoperative drainage and faster healing. However prolonged application of can cause excessive damage due to slowly progressing liquefaction necrosis [17].

Trichloroacetic acid is one of the most commonly used agents for chemical peeling. Depending on the concentration, it achieves superficial to medium depth chemical peeling. It is a caustic chemical agent that causes coagulation necrosis, like phenol. It produces both epidermal and dermal necrosis and then neutralizes by itself without serious systemic toxicity. In a recent study, Kim et al. performed chemical matricectomy with $100 \%$ TCA in 25 patients with ingrowing toenail edges, and reported that the success rate was $95 \%$. They reported that adverse effects such as postoperative pain, drainage and infection were mild; postoperative drainage generally decreased within one week and did not last more than two weeks [15].

Other methods used are surgical matricectomy and ablative methods such as carbon dioxide laser [15,17], electrocautery and radiofrequency [18-20]. The latter techniques are more technically demanding and more expensive compared with chemical matricectomy, and hence not used as commonly.

Previous studies have compared sodium hydroxide and phenol but had differing results [2,14]. However we didn't find any study comparing TCA to Phenol. Our study shows that: the two techniques studied allow good results in terms of recidivism and aesthetic result. On the other hand one can obtain a faster cicatrization and avoid the patients the postoperative oozing by using the TCA.

\section{CONCLUSION}

The TCA method is also fast, easy to perform, and with less postoperative oozing with faster healing. The destruction by TCA is therefore considered as a technique of choice and allows good aesthetic results with a low recurrence rate as evidenced by our study. To our knowledge, this is the first study comparing these two methods, and other studies are needed to confirm our results and to adopt this technique of matricolysis.

\section{Statement of Human and Animal Rights}

All procedures followed were in accordance with the ethical standards of the responsible committee on human experimentation (institutional and national) and with the Helsinki Declaration of 1975, as revised in 2008.

\section{Statement of Informed Consent}

Informed consent was obtained from all patients for being included in the study.

\section{REFERENCES}

1. Camurcu Y, Sofu H, Issin A, Kockara N, Saygili H. Operative treatment of the ingrown toenail with a less-invasive technique: flashback to the original winograd technique.foot ankle spec. 2018;11:138-41.

2. Tatlican S, Eren C, Yamangokturk B, Eskioglu F. Letter: Retrospective comparison of experiences with phenol and sodium hydroxide in the treatment of ingrown nail. Dermatol Surg. 2010;36:432-4.

3. Zaraa I, Dorbani I, Hawilo A, Mokni M, Ben Osman A. Segmental phenolization for the treatment of ingrown toenails: technique report, follow up of 146 patients, and review of the literature. Dermatol Online J. 2013;19:18560.

4. Terzi E, Guvenc U, Türsen B, Kaya TI, Erdem T, Türsen Ü. The effectiveness of matrix cauterization with trichloroacetic acid in the treatment of ingrown toenails.Indian Dermatol Online J. 2015;6:4-8.

5. Bostanci S, Kocyigit P, Parlak N, Gungor HK. Chemical matricectomy with sodium hydroxide: long-term followup results. Dermatol Surg. 2014;40:1221-4.

6. Tatlican S, Yamangöktürk B, Eren C, Eskioğlu F, Adiyaman S. Comparison of phenol applications of different durations for the cauterization of the germinal matrix: An efficacy and safety study. Acta Orthop Traumatol Turc. 2009;43:298-302.

7. Eekhof JA, Van Wijk B, Knuistingh Neven A, van der Wouden JC. Interventions for ingrowing toenails. Cochrane Database Syst Rev. 2012;4:CD001541.

8. Vural S, Bostanci S, Koçyigit P, Çaliskan D, Baskal N, Aydin N. Risk Factors and Frequency of Ingrown Nails in Adult Diabetic Patients. J Foot Ankle Surg. 2018;57:289-95.

9. Jia C, Li P, Wu Y, Qiu Y, Cao L, Chang C, et al. Modified surgical repair of severe ingrown toenail. Zhongguo Xiu Fu Chong Jian Wai Ke Za Zhi. 2013;27:869-71.

10. Karaca N, Dereli T. Treatment of ingrown toenail with proximolateral matrix partial excision and matrix phenolization. Ann Fam Med. 2012;10:556-9.

11. Vaccari S, Dika E, Balestri R, Rech G, Piraccini BM, Fanti PA. 
Partial excision of matrix and phenolic ablation for the treatment of ingrowing toenail: a 36-month follow-up of 197 treated patients. Dermatol Surg. 2010;36:1288-93.

12. Di Chiacchio N, Di Chiacchio NG. Best way to treat an ingrown toenail. Dermatol Clin. 2015;33:277-82.

13. Chiacchio ND, Belda W Jr, Chiacchio NG, Kezam Gabriel FV, de Farias DC. Nail matrix phenolization for treatment of ingrowing nail: Technique report and recurrence rate of 267 surgeries. Dermatol Surg. 2010;36:534-7.

14. Richert B. Surgical management of ingrown toenails - an update overdue. Dermatol Ther. 2012;25:498-509.

15. Becerro de Bengoa Vallejo R, Cordoba Diaz D, Cordoba Diaz M, Losa Iglesias ME. Alcohol irrigation after phenol chemical matricectomy: an in vivo study. Eur J Dermatol. 2013;23:319-23.

16. Eekhof JA, Van Wijk B, Knuistingh Neven A, van der Wouden JC. Interventions for ingrowing toenails.Cochrane Database Syst Rev. 2012;(4):CD001541.

17. Pérez-Rey J, Mediavilla-Saldaña L, Martínez-Nova A. Exploring postoperative outcomes for ingrown toenails. $\mathrm{NaOH}$ vs wedge resection techniques. Dermatol Surg. 2014;40:281-7.

18. Becerro de Bengoa Vallejo R, Losa Iglesias ME, Viejo Tirado F, Serrano Pardo R. Cauterization of the germinal nail matrix using phenol applications of differing durations: A histologic study. J Am Acad Dermatol. 2012;67:706-11.

19. Cocunubo-Blanco HA, González-Sixto B, Pérez-Paredes G, Rodríguez-Prieto MÁ. Partial nail matricectomy with carbon dioxide laser. Actas Dermosifiliogr. 2014;105:418-9.

20. Heidelbaugh JJ, Lee H. Management of the ingrown toenail. Am Fam Physician. 2009;79:303-8.

Copyright by Kaoutar Moustaide, et al. This is an open-access article distributed under the terms of the Creative Commons Attribution License, which permits unrestricted use, distribution, and reproduction in any medium, provided the original author and source are credited.

Source of Support: Nil, Conflict of Interest: None declared. 